Arq. Bras. Med. Vet. Zootec., v.67, n.5, p.1361-1371, 2015

\title{
Crescimento de genótipos de frangos tipo caipira
}

\author{
[Growth of genotypes of an alternative strain of broiler chickens] \\ R.C. Veloso ${ }^{1}$, A.V. Pires ${ }^{1}$, R.A. Torres Filho ${ }^{2}$, E.C.S. Drumond ${ }^{1}$, L.S. Costa ${ }^{1}$, \\ J.M. Amaral ${ }^{1}$, I.G. Pereira ${ }^{3}$ \\ ${ }^{1}$ Universidade Federal dos Vales do Jequitinhonha e Mucuri - Diamantina, MG \\ ${ }^{2}$ Universidade Federal Fluminense - Niterói, RJ \\ ${ }^{3}$ Universidade Federal de Minas Gerais - Belo Horizonte, MG
}

\begin{abstract}
RESUMO
Objetivou-se com este trabalho comparar o padrão de crescimento, mediante ajustes das respectivas curvas de crescimento por modelos não lineares, bem como estudar o desenvolvimento de cortes de carcaça em relação ao peso da carcaça em diferentes genótipos de frangos tipo caipira. Foram utilizados 840 pintos de um dia, machos, distribuídos em delineamento inteiramente ao acaso, dos seguintes genótipos da linhagem Redbro: Caboclo, Carijó, Colorpak, Gigante Negro, Pesadão Vermelho, Pescoço Pelado e Tricolor. As aves foram alojadas em 28 boxes, sendo 30 aves/boxe, em galpão de alvenaria com acesso a um piquete de $45 \mathrm{~m}^{2}$, com quatro repetições. $\mathrm{O}$ peso corporal individual dos frangos foi medido ao nascer, aos 14, 28, 42, 56, 70 e 84 dias de idade. Para a determinação das curvas de crescimento do peso corporal das aves, os dados coletados foram avaliados por meio dos modelos não lineares: Brody, Gompertz, Logístico, Richards e von Bertalanffy. Foi empregado o PROC NLIN do SAS, utilizando-se o método interativo de Gauss-Newton. Os critérios usados para escolha do modelo de melhor ajuste da curva de crescimento foram o coeficiente de determinação, o desvio padrão assintótico, o desvio médio absoluto dos resíduos e o índice assintótico. As análises para obtenção dos coeficientes alométricos foram realizadas por meio do PROC GLM do SAS para os genótipos Carijó, Colorpak, Pesadão Vermelho, Pescoço Pelado e Tricolor. Foram avaliados os pesos da carcaça, do peito, das coxas, das sobrecoxas, das pernas e das asas das aves abatidas aos 85 dias de idade. Apenas as equações propostas por Gompertz, von Bertalanffy e Logístico atingiram a convergência, e o modelo proposto por von Bertalanffy foi o mais adequado para descrever o crescimento dos genótipos de frangos caipiras. Todos os cortes avaliados apresentaram crescimento tardio em relação ao peso da carcaça em genótipos de frangos tipo caipira.
\end{abstract}

Palavras-chave: Gompertz, índice assintótico, Logístico, von Bertalanffy

\begin{abstract}
The aim of this work was to compare the growth pattern, adjusting the growth curves by nonlinear models and studying the development of housing sections in relation to the weight of the chicken carcass in different genotypes of an alternative strain of broiler chickens. We used 840 one day old male chicks distributed in a completely randomized design, with the following genotype strains: Redbro: Caboclo, Carijó, Colorpak, Gigante Negro, Pesadão Vermelho, Pescoço Pelado and Tricolor. The birds were housed in 28 boxes, 30 birds / box in a masonry shed with access to a 45 square meter paddock, with four replications. Body weight was measured in chickens up to 84 days of age. To determine the growth curves of the body weight of birds, collected data were evaluated from the nonlinear models: Brody, Gompertz, Logistic, Richards and von Bertalanffy. Was used "proc nlin" of SAS, using the iterative method of Gauss-Newton. The criteria used to choose the best model for the growth curve were the coefficient of determination, the asymptotic standard deviation, the mean absolute deviation of the waste and the asymptotic index. The analysis to obtain the allometric coefficients were performed using the "proc glm" SAS for genotypes Carijó, Colorpak, Pesadão Vermelho, Pescoço Pelado and Tricolor. We assessed the
\end{abstract}

Recebido em 29 de abril de 2013

Aceito em 4 de maio de 2015

E-mail: velosozootecnista@yahoo.com.br 
weights of carcass, breast, thighs, drumsticks, legs and wings. Only the equations proposed by Gompertz, Logistic and von Bertalanfy reached convergence, and the model proposed by von Bertalanffy was the most appropriate. All sections were evaluated in relation to the late growth of carcass weight in alternative lines of broiler chickens.

Keywords: asymptotic index, Gompertz, Logistic, von Bertalanffy

\section{INTRODUÇÃO}

Trabalhos na área de genética vêm sendo realizados com o objetivo de desenvolver aves mais adaptadas, a fim de melhorar os índices produtivos da criação alternativa (Silva et al., 2002). Nesse sentido, é de extrema importância conhecer o padrão e o potencial de crescimento das aves, expresso em peso corporal e ganho de peso.

Esse desenvolvimento dos animais pode ser representado por curvas de crescimento ajustadas por polinômios descritos por funções logarítmicas e pode ser dividido em três fases: a primeira, $\log$ positiva, a segunda, log negativa, e a terceira, estacionária. As duas primeiras definem o crescimento exponencial, crescente e decrescente, respectivamente (Tholon e Queiroz, 2009).

Os modelos matemáticos não lineares podem ser utilizados como instrumentos para descrever o desenvolvimento e o crescimento da carcaça e das partes que permitem uma análise, a fim de se adotarem estratégias que possibilitem melhores desempenhos, principalmente no que se refere ao aumento do ganho de peso e da eficiência alimentar. Para frangos de corte, parâmetros de modelos não lineares estimados para o crescimento podem oferecer informações para se selecionarem algumas características de carcaça associadas à melhor idade de abate e para se minimizarem as perdas provocadas pelas doenças metabólicas oriundas do rápido crescimento. Além disso, podem fornecer subsídio para o setor avícola e, assim, por meio da estimativa do crescimento das aves, determinar as exigências nutricionais, predizer a melhor idade de abate, predizer índices de eficiência de produção, bem como proporcionar dados para desenvolver modelos de crescimento (Marcato et al., 2010).

Dessa forma, torna-se necessária a avaliação de genótipos tipo caipira disponíveis para criação em sistema semi-intensivo no que se refere às informações relacionadas ao crescimento e ao desempenho desses genótipos, que são importantes para o aumento da lucratividade desse sistema de produção.

Considerando esses aspectos, objetivou-se com este trabalho comparar o padrão de crescimento, mediante ajustes das respectivas curvas de crescimento por modelos não lineares, bem como estudar o desenvolvimento de cortes de carcaça em relação ao peso da carcaça em diferentes genótipos de frangos tipo caipira.

\section{MATERIAL E MÉTODOS}

O experimento foi conduzido no setor de Avicultura do Departamento de Zootecnia da Universidade Federal dos Vales do Jequitinhonha e Mucuri, situado no Campus JK em Diamantina-MG, durante o período de 21 de fevereiro a 16 de maio de 2011.

Foram utilizados 840 pintos de um dia, machos, provenientes de sete genótipos de frangos tipo caipira da linhagem Redbro: Caboclo (CBC), Carijó (CG), Colorpak (CPK), Gigante Negro (GNG), Pesadão Vermelho (PS), Pescoço Pelado (PP) e Tricolor (TRC). Os animais foram alojados em 28 boxes de dimensões 2,00 x 2,00m em galpão de alvenaria, coberto por telhas de fibrocimento, sendo 30 aves/boxe em quatro repetições para cada genótipo. Aos 28 dias, os animais de cada boxe tiveram acesso a um piquete de $30 \mathrm{~m}^{2}$, formado por forrageira do tipo Tifton, gênero Cynodon.

O peso corporal individual dos frangos foi medido ao nascer, aos $14,28,42,56,70$ e 84 dias de idade.

As rações utilizadas foram formuladas à base de milho e farelo de soja, para as diferentes fases: inicial (um a 28 dias), crescimento ( 28 a 56 dias) e final (56 a 84 dias) (Tab. 1), conforme informações de composições e digestibilidade dos ingredientes apresentados em Rostagno et al. 
(2005); Mendonça et al. (2008); Nagib Nascimento et al. (2009a;b); Pinheiro et al. (2011a;b).

As rações foram fornecidas à vontade, 24 horas por dia, até os 70 dias. Após esse período, os animais passaram por restrição alimentar quantitativa, quando receberam ração à vontade por apenas sete horas ao dia, das nove às 16 horas.

Para a determinação das curvas de crescimento do peso corporal das aves, os dados coletados foram avaliados por meio dos modelos de crescimento:

$$
\begin{aligned}
& \text { Gompertz: } y=a \exp (-b \exp (-k t)) \text {; } \\
& \text { Brody: } y=a(1-b \exp (-k t)) ; \\
& \text { Logistico: } y=a\left(1+\exp (-k t)^{-\mathrm{m}}\right) ;
\end{aligned}
$$

von Bertalanffy: $y=a\left(1-b \exp (-k t)^{2}\right)$; Ritchards: $y=a\left(1-b \exp (-k t)^{m}\right)$,

em que: "y" é o peso corporal na idade "t"; "a" é o peso assintótico quando " $\mathrm{t}$ " tende a mais infinito, ou seja, interpretado como peso à idade adulta; e "b" é uma constante de integração, relacionada aos pesos iniciais do animal e sem interpretação biológica bem definida. $\mathrm{O}$ valor de " $\mathrm{k}$ " é estabelecido pelos valores iniciais de " $\mathrm{y}$ " e "t"; " $k$ " é interpretado como a taxa de maturação, que deve ser entendida como a mudança no peso corporal em relação ao peso à maturidade, ou seja, o indicador da velocidade com que o animal se aproxima do seu tamanho adulto, ou como a taxa de crescimento relativo ao peso máximo; e "m" é o parâmetro que dá forma à curva: sua fixação determina a forma da curva e,

\begin{tabular}{|c|c|c|c|}
\hline Ingredientes (\%) & 1 a 28 dias & 28 a 56 dias & 56 a 84 dias \\
\hline Milho moído & 56,792 & 62,518 & 71,793 \\
\hline Farelo de soja $45 \%$ & 36,302 & 30,822 & 24,089 \\
\hline Inerte (areia fina) & 2,558 & 3,000 & 0,914 \\
\hline Fosfato bicálcico & 2,044 & 1,327 & 1,212 \\
\hline Calcário & 1,429 & 1,196 & 1,094 \\
\hline Sal comum & 0,457 & 0,331 & 0,254 \\
\hline DL- metionina & 0,217 & 0,174 & 0,260 \\
\hline Suplemento vitamínico ${ }^{1}$ & 0,100 & 0,100 & 0,100 \\
\hline Suplemento mineral ${ }^{2}$ & 0,100 & 0,100 & 0,100 \\
\hline Óleo de soja & 0,000 & 0,311 & 0,184 \\
\hline L- Lisina HCL & 0,000 & 0,120 & 0,000 \\
\hline Total & 100,00 & 100,00 & 100,00 \\
\hline \multicolumn{4}{|l|}{ Composição química calculada } \\
\hline Proteína bruta $(\%)$ & 21,27 & 19,35 & 17,00 \\
\hline Energia metabolizável (kcal/kg) & 2750 & 2850 & 3000 \\
\hline Lisina digestível (\%) & 1,04 & 1,00 & 0,76 \\
\hline Metionina digestível (\%) & 0,51 & 0,44 & 0,50 \\
\hline Metionina + cistina digestível $(\%)$ & 0,80 & 0,71 & 0,75 \\
\hline Cálcio $(\%)$ & 1,16 & 0,88 & 0,80 \\
\hline Fósforo disponível (\%) & 0,48 & 0,35 & 0,32 \\
\hline
\end{tabular}
consequentemente, o ponto de inflexão.

Tabela 1. Composições das rações nas três fases de criação das aves

${ }^{\mathrm{I}}$ Suplemento vitamínico por kg de produto: vit. A 3.750.000UI; vit. $\mathrm{D}_{3}, 750.000 \mathrm{UI}$; vit. E $7500 \mathrm{mg}$; vit $\mathrm{K}_{3}, 1.000 \mathrm{mg}$; vit. $\mathrm{B}_{1}, 750 \mathrm{mg}$; vit. $\mathrm{B}_{2}, 1.500 \mathrm{mg}$; vit. $\mathrm{B}_{6}, 1500 \mathrm{mg}$; vit. $\mathrm{B}_{12}, 7.500 \mathrm{mcg}$; vit. C $12.500 \mathrm{mg}$; biotina $30 \mathrm{mg}$; niacina $10.000 \mathrm{mg}$; ácido fólico 375 ; ácido pantotênico $3.750 \mathrm{mg}$; colina $10.000 \mathrm{mg}$; metionina $400.000 \mathrm{mg}$.

${ }^{2}$ Suplemento mineral por kg de produto: selênio $45 \mathrm{mg}$; iodo $175 \mathrm{mg}$; ferro $12.525 \mathrm{mg}$; cobre $2.500 \mathrm{mg}$; manganês 19.500mg; zinco 13.750mg; prom. Prod $15.000 \mathrm{mg}$; coccidiostático $10.000 \mathrm{mg}$; antioxidante (BHT) 500mg.

As análises estatísticas foram realizadas utilizando-se o procedimento para análise de modelos não lineares (PROC NLIN) do software Statistical Analysis System (SAS, 2002), por meio do método iterativo de Gauss-Newton. Os critérios usados para escolha do modelo de melhor ajuste da curva de crescimento foram o coeficiente de determinação $\left(\mathrm{R}^{2}\right)$, o 
desvio padrão assintótico (DPA), o desvio médio absoluto (DMA) e o índice assintótico (IA). $\mathrm{O} \quad \mathrm{R}^{2}$ foi calculado pela função $1-\left(\$ Q R / S Q T_{C}\right)$, sendo $\mathrm{SQR}$ a soma de quadrados do resíduo e SQTc a soma de quadrados total corrigida pela média; o desvio padrão assintótico (DPA) foi calculado pela raiz quadrada do quadrado médio do resíduo de cada modelo; e o desvio médio absoluto dos resíduos (DMA), conforme proposto por Sarmento et al. (2006), foi calculado como a seguir:

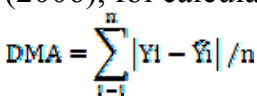

O índice assintótico (IA), conforme descrito por Ratkowsky (1990), foi calculado mediante a combinação dos critérios DPA, DMA e $\mathrm{R}^{2}$. Atribui-se valor 100 para a maior estimativa de cada critério, e os demais foram ponderados em relação a este; assim: $\mathrm{IA}=(\mathrm{DPA}+\mathrm{DMA})-\mathrm{R}^{2}$. Quanto menor o índice, melhor o ajuste do modelo.

Para o estudo de crescimento alométrico, foram abatidas oito aves de cada genótipo: Carijó, Colorpak, Pesadão Vermelho, Pescoço Pelado e Tricolor, ao nascimento, $14,28,42,56,70$ e 84 dias de idade, em um total de 280 aves, em que foram avaliados os pesos da carcaça (PCA), do peito (PP), das coxas (PC), das sobrecoxas (PSC), das pernas (PPER) e das asas (PA).

O estudo do crescimento relativo dos cortes foi realizado mediante o modelo da equação alométrica de Huxley $\left(y=a x^{2}\right)$ transformada logaritmicamente em um modelo linear, $\ln Y=\ln a+b \ln X$ (Huxley, 1932), em que: " $y$ " é o peso do corte; " $x$ " é o peso da carcaça; " $a$ " é o intercepto; " $b$ " é o coeficiente de crescimento relativo ou de alometria.

As análises para obtenção dos coeficientes alométricos foram realizadas por meio do PROC GLM do SAS (2002). Para verificação da hipótese $\mathrm{b}=1$, foi realizado o teste $t$ (Student) $(\mathrm{P}<0,01)$ para as diferenças entre genótipo $\mathrm{e}$ idade de abate.

Quando $\mathrm{b}=1$, o crescimento foi denominado isogônico, indicando que as taxas de desenvolvimento de "x" e "y" são semelhantes no intervalo de crescimento considerado. Quando $b$ $\neq 1$, o crescimento foi chamado heterogônico; se positivo (b>1), o órgão é considerado de desenvolvimento tardio, se negativo $(b<1)$, de desenvolvimento precoce.

\section{RESULTADOS E DISCUSSÃO}

Verificou-se que todos os genótipos estudados atingiram o peso corporal preconizado para o abate $(2300 \mathrm{~g})$ aos 70 dias, conforme foi publicado no Ofício Circular DIPOA N 02/2012 (Mapa, 2012), reduzindo, assim, a idade de abate e, consequentemente, o custo de produção da criação de frangos tipo caipira (Tab. 2).

Tabela 2. Média dos pesos corporais (g) em diferentes idades (um, 28, 56, 70 e 84 dias), para os genótipos Caboclo, Carijó, Colorpak, Gigante Negro, Pesadão Vermelho, Pescoço Pelado e Tricolor

\begin{tabular}{lccccccc}
\hline \multirow{2}{*}{ Genótipo } & \multicolumn{7}{c}{ Peso corporal $(\mathrm{g})$} \\
\cline { 2 - 7 } & 1 dia & 14 dias & 28 dias & 42 dias & 56 dias & 70 dias & 84 dias \\
\hline Caboclo & 38,31 & 223,39 & 633,27 & 1161,21 & 1837,84 & 2369,80 & 2910,53 \\
Carijó & 33,55 & 299,65 & 953,99 & 1830,67 & 2791,69 & 3299,90 & 3850,73 \\
Colorpak & 40,00 & 315,91 & 986,34 & 1925,21 & 3057,78 & 3645,96 & 4227,97 \\
Gigante Negro & 39,69 & 232,65 & 686,12 & 1245,61 & 1962,08 & 2458,89 & 3002,49 \\
Pesadão Vermelho & 33,91 & 289,50 & 928,37 & 1775,67 & 2795,75 & 3342,22 & 3915,36 \\
Pescoço Pelado & 41,33 & 306,68 & 906,14 & 1686,69 & 2606,88 & 3097,79 & 3637,56 \\
Tricolor & 40,87 & 304,39 & 944,11 & 1760,50 & 2719,48 & 3283,73 & 3912,60 \\
\hline
\end{tabular}

Apenas as equações propostas por von Bertalanffy, Gompertz e Logístico atingiram a convergência (Tab. 3, 4 e 5, respectivamente), enquanto as análises realizadas pelos modelos Richards e Brody não convergiram, mostrando que estes modelos não se adequaram à descrição dos pesos para os genótipos estudados.

Para o modelo von Bertalanffy, o parâmetro "a" (peso à idade adulta) apresentou maior valor para 
o Colorpak e menor valor para o Pescoço Pelado (Tab. 3). Esse resultado evidencia que as aves do genótipo Colorpak tendem a apresentar o maior peso à idade adulta e as aves do genótipo Pescoço Pelado, o menor peso à maturidade. Para todos os genótipos, os valores de "a" foram superestimados em relação ao peso médio dos animais aos 84 dias (Tab. 3), o que mostra que todos os genótipos, ao final do experimento, não haviam atingido seu peso máximo, ou seja, ainda estavam em fase de crescimento e deposição muscular.

O parâmetro "b", constante de integração, não possui interpretação biológica e, portanto, não será discutido.
O parâmetro " $k$ " representa a velocidade com que o animal passa pelo período de crescimento até se tornar adulto. No modelo von Bertalanffy, o Carijó apresentou maior valor de "k", e o Caboclo o menor valor, evidenciando que os animais Carijó apresentam maior taxa de crescimento e os Caboclo, menor taxa para esse modelo. O Caboclo foi o que obteve menor valor (melhor ajuste) do desvio médio absoluto (DMA), o Colorpak foi o que apresentou maior (pior ajuste) valor de DMA.

Em relação ao desvio padrão assintótico (DPA), observou-se que os genótipos Carijó, Colorpak e Pesadão Vermelho obtiveram os maiores valores (pior ajuste), e o Caboclo obteve o menor valor (melhor ajuste).

Tabela 3. Parâmetros estimados para as curvas de crescimento dos genótipos de frangos tipo caipira segundo o modelo von Bertalanffy

\begin{tabular}{|c|c|c|c|c|c|c|c|}
\hline \multirow{2}{*}{ Genótipo } & \multicolumn{3}{|c|}{ Parâmetro } & \multirow{2}{*}{$\begin{array}{l}\mathrm{R}^{2} \\
(\%)\end{array}$} & \multirow{2}{*}{$\begin{array}{c}\text { DMA } \\
(\%)\end{array}$} & \multirow{2}{*}{$\begin{array}{c}\text { DPA } \\
(\%)\end{array}$} & \multirow{2}{*}{ IA } \\
\hline & $\mathrm{a}$ & $\mathrm{b}$ & $\mathrm{k}$ & & & & \\
\hline \multirow[b]{2}{*}{ Caboclo } & 5245,9 & 0,8493 & 0,0186 & \multirow[b]{2}{*}{98,42} & \multirow[b]{2}{*}{26,14} & \multirow[b]{2}{*}{19,25} & \multirow[b]{2}{*}{53,03} \\
\hline & $\begin{array}{c}(4908,3 \mathrm{a} \\
5583,5)^{1}\end{array}$ & $\begin{array}{c}(0,8309 \mathrm{a} \\
0,8677)\end{array}$ & $\begin{array}{c}(0,0173 \mathrm{a} \\
0,0199)\end{array}$ & & & & \\
\hline \multirow[b]{2}{*}{ Carijó } & 4984,0 & 0,9374 & 0,0289 & \multirow[b]{2}{*}{95,99} & \multirow[b]{2}{*}{74,51} & \multirow[b]{2}{*}{90,94} & \multirow[b]{2}{*}{69,46} \\
\hline & $\begin{array}{c}(4777,1 \mathrm{a} \\
5190,9)\end{array}$ & $\begin{array}{c}(0,8973 \mathrm{a} \\
0,9775) \\
\end{array}$ & $\begin{array}{c}(0,0270 \mathrm{a} \\
0,0308)\end{array}$ & & & & \\
\hline \multirow[b]{2}{*}{ Colorpak } & 5585,5 & 0,9604 & 0,0286 & \multirow[b]{2}{*}{96,36} & \multirow[b]{2}{*}{100,00} & \multirow[b]{2}{*}{100,00} & \multirow[b]{2}{*}{103,64} \\
\hline & $\begin{array}{c}(5351,6 \mathrm{a} \\
5819,4)\end{array}$ & $\begin{array}{c}(0,9199 \mathrm{a} \\
1,0009)\end{array}$ & $\begin{array}{c}(0,0267 \mathrm{a} \\
0,0304)\end{array}$ & & & & \\
\hline \multirow[b]{2}{*}{ Gigante Negro } & 4960,7 & 0,8532 & 0,0203 & \multirow[b]{2}{*}{95,80} & \multirow[b]{2}{*}{37,39} & \multirow[b]{2}{*}{55,70} & \multirow[b]{2}{*}{2,71} \\
\hline & $\begin{array}{c}(4503,9 \mathrm{a} \\
5417,6)\end{array}$ & $\begin{array}{c}(0,8196 \mathrm{a} \\
0,8868) \\
\end{array}$ & $\begin{array}{c}(0,0181 \mathrm{a} \\
0,0225)\end{array}$ & & & & \\
\hline \multirow[b]{2}{*}{$\begin{array}{l}\text { Pesadão } \\
\text { Vermelho }\end{array}$} & 5245,7 & 0,9390 & 0,0276 & \multirow[b]{2}{*}{95,87} & \multirow[b]{2}{*}{79,52} & \multirow[b]{2}{*}{95,89} & \multirow[b]{2}{*}{79,53} \\
\hline & $\begin{array}{c}(5001,4 \mathrm{a} \\
5489.9)\end{array}$ & $\begin{array}{c}(0,8988 \mathrm{a} \\
0,9792) \\
\end{array}$ & $\begin{array}{l}(0,0257 \mathrm{a} \\
0,0296)\end{array}$ & & & & \\
\hline \multirow[b]{2}{*}{ Pescoço Pelado } & 4922,7 & 0,8969 & 0,0266 & \multirow[b]{2}{*}{95,84} & \multirow[b]{2}{*}{72,23} & \multirow[b]{2}{*}{82,16} & \multirow[b]{2}{*}{58,55} \\
\hline & $\begin{array}{c}(4688,2 \mathrm{a} \\
5157,2)\end{array}$ & $\begin{array}{c}(0,8617 \mathrm{a} \\
0,9320) \\
\end{array}$ & $\begin{array}{c}(0,0248 \mathrm{a} \\
0,0285)\end{array}$ & & & & \\
\hline Tricolor & $\begin{array}{c}5504,3 \\
(5247,8 \mathrm{a} \\
5760,9) \\
\end{array}$ & $\begin{array}{c}0,8914 \\
(0,8619 \mathrm{a} \\
0,9210)\end{array}$ & $\begin{array}{c}0,0251 \\
(0,0235 \mathrm{a} \\
0,0268)\end{array}$ & 96,76 & 64,03 & 72,15 & 39,42 \\
\hline
\end{tabular}

a-peso à idade adulta; b-constante de integração; k-taxa de maturidade; $\mathrm{R}^{2}$-coeficiente de determinação; DMA-desvio médio absoluto; DPA-desvio padrão assintótico; IA- índice assintótico.

${ }^{1}$ Intervalo de confiança a $95 \%$ entre parênteses.

O menor valor para o índice assintótico (IA) foi observado para o Gigante Negro, indicando que o modelo von Bertalanffy foi o que apresentou o melhor ajuste para esse genótipo. Haja vista que o IA é um critério que combina $\mathrm{R}^{2}$, DPA e DMA, em princípio é considerado um critério "mais completo", sendo, portanto, o recomendado para a escolha de modelos (Araújo et al., 2012). 
Dessa forma, o modelo von Bertalanffy apresentou, em termos gerais, o melhor ajuste para o Gigante Negro e o pior ajuste para o Colorpak.

Para o modelo Gompertz, pode ser observado que o maior valor do parâmetro "a" foi obtido pelo Colorpak, o que indica que essas aves apresentaram maior peso à idade adulta. $\mathrm{O}$ Gigante Negro apresentou menor valor para esse mesmo parâmetro, mostrando, assim, que são aves mais tardias (Tab. 4). Ainda nesse modelo, o Carijó obteve maior valor de "k", e o Caboclo o menor valor. Sendo "k" a velocidade com que o animal passa pelo período de crescimento até se tornar adulto, percebe-se que o Carijó possui maior velocidade de crescimento em relação aos demais genótipos estudados.

Em trabalho realizado por Santos et al. (2005) com as linhagens caipiras Paraíso Pedrês e Pescoço Pelado, a Paraíso Pedrês apresentou maior potencial de crescimento, estimado pelo peso à maturidade, maior taxa de maturidade $\mathrm{e}$ menor idade para máximo crescimento que a Pescoço Pelado, utilizando-se o modelo Gompertz.

Considerando os critérios para qualidade de ajuste do modelo (DMA, DPA e IA) observou-se que o genótipo Caboclo obteve menores valores e o Colorpak os maiores valores.

Tabela 4. Parâmetros estimados para curvas de crescimento dos genótipos de frangos tipo caipira segundo o modelo Gompertz

\begin{tabular}{|c|c|c|c|c|c|c|c|}
\hline \multirow{2}{*}{ Genótipo } & \multicolumn{3}{|c|}{ Parâmetro } & \multirow{2}{*}{$\begin{array}{l}\mathrm{R}^{2} \\
(\%)\end{array}$} & \multirow{2}{*}{$\begin{array}{c}\text { DMA } \\
(\%)\end{array}$} & \multirow{2}{*}{$\begin{array}{c}\text { DPA } \\
(\%)\end{array}$} & \multirow{2}{*}{ IA } \\
\hline & $\mathrm{a}$ & $\mathrm{b}$ & $\mathrm{k}$ & & & & \\
\hline \multirow[b]{2}{*}{ Caboclo } & 4150,4 & 4,3888 & 0,0298 & \multirow[b]{2}{*}{98,42} & \multirow[b]{2}{*}{49,42} & \multirow[b]{2}{*}{19,76} & \multirow[b]{2}{*}{29,25} \\
\hline & $\begin{array}{c}(3986,4 \mathrm{a} \\
4314,4)^{1}\end{array}$ & $(4,24$ a 4,53) & $\begin{array}{c}(0,028 \mathrm{a} \\
0,031)\end{array}$ & & & & \\
\hline \multirow[b]{2}{*}{ Carijó } & 4487,6 & 4487,6 & 0,0404 & \multirow[b]{2}{*}{96,03} & \multirow[b]{2}{*}{86,63} & \multirow[b]{2}{*}{92,23} & \multirow[b]{2}{*}{82,84} \\
\hline & $\begin{array}{c}(4356,0 \mathrm{a} \\
4619,2)\end{array}$ & $(4,53$ a 5,072$)$ & $\begin{array}{c}(4,538 \mathrm{a} \\
5,072)\end{array}$ & & & & \\
\hline \multirow[b]{2}{*}{ Colorpak } & 4995,9 & 5,0101 & 0,0403 & \multirow[b]{2}{*}{96,43} & \multirow[b]{2}{*}{100,00} & \multirow{2}{*}{$\begin{array}{c}100,0 \\
0\end{array}$} & \multirow{2}{*}{$\begin{array}{c}103,5 \\
7\end{array}$} \\
\hline & $\begin{array}{c}(4850,5 \mathrm{a} \\
5141.3)\end{array}$ & $(4,73$ a 5,284$)$ & $\begin{array}{c}(0,038 \mathrm{a} \\
0,042)\end{array}$ & & & & \\
\hline \multirow[b]{2}{*}{$\begin{array}{l}\text { Gigante } \\
\text { Negro }\end{array}$} & 4067,3 & 4,3767 & 0,0315 & \multirow[b]{2}{*}{73,11} & \multirow[b]{2}{*}{71,61} & \multirow[b]{2}{*}{57,11} & \multirow[b]{2}{*}{55,61} \\
\hline & $\begin{array}{c}(3829,4 \mathrm{a} \\
4305,1)\end{array}$ & $\begin{array}{c}(4,1278 \mathrm{a} \\
4,6256)\end{array}$ & $\begin{array}{c}(0,0290 \mathrm{a} \\
0,0339)\end{array}$ & & & & \\
\hline \multirow{2}{*}{$\begin{array}{l}\text { Pesadão } \\
\text { Vermelho }\end{array}$} & 4662,1 & 4,8695 & 0,0392 & \multirow[b]{2}{*}{95,92} & \multirow[b]{2}{*}{96,25} & \multirow[b]{2}{*}{96,98} & \multirow[b]{2}{*}{97,31} \\
\hline & $\begin{array}{c}(4511,9 \mathrm{a} \\
4812,4) \\
\end{array}$ & $\begin{array}{c}(4,5966 \mathrm{a} \\
5,142)\end{array}$ & $\begin{array}{l}(0,0371 \mathrm{a} \\
0,0414)\end{array}$ & & & & \\
\hline \multirow[b]{2}{*}{$\begin{array}{l}\text { Pescoço } \\
\text { Pelado }\end{array}$} & 4353,1 & 4,5785 & 0.0381 & \multirow[b]{2}{*}{94,69} & \multirow[b]{2}{*}{80,53} & \multirow[b]{2}{*}{83,22} & \multirow[b]{2}{*}{69,06} \\
\hline & $\begin{array}{c}(4210,9 \mathrm{a} \\
4495,4)\end{array}$ & $\begin{array}{c}(4,3402 \mathrm{a} \\
4,8168)\end{array}$ & $\begin{array}{c}(0,0360 \mathrm{a} \\
0,0402)\end{array}$ & & & & \\
\hline \multirow[b]{2}{*}{ Tricolor } & 4792,8 & 4,5703 & 0,0365 & & & & \\
\hline & $\begin{array}{c}(4641,8 \mathrm{a} \\
4943,7)\end{array}$ & $\begin{array}{c}(4,3664 \mathrm{a} \\
4,7742)\end{array}$ & $\begin{array}{c}(0,0347 \mathrm{a} \\
0,0383)\end{array}$ & 96,77 & 94,40 & 73,56 & 71,19 \\
\hline
\end{tabular}

a-peso à idade adulta; b-constante de integração; k-taxa de maturidade; $\mathrm{R}^{2}$-coeficiente de determinação; DMA-desvio médio absoluto; DPA-desvio padrão assintótico; IA- índice assintótico.

${ }^{1}$ Intervalo de confiança a $95 \%$ entre parênteses.

O melhor ajuste da curva é atingido quando se obtêm menores valores de DMA, DPA e IA; assim, pode-se afirmar que o padrão de crescimento das aves do genótipo Caboclo se ajustou bem ao modelo Gompertz.
Pela avaliação do modelo Logístico, assim como do modelo Gompertz, as aves do genótipo Colorpak obtiveram maior valor para o parâmetro "a", e as do Gigante Negro menor valor (Tab. 5). 
Como também observado em outros modelos (von Bertalanffy e Gompertz), os valores de "a" de todos os genótipos foram superestimados em relação ao peso médio dos animais aos 84 dias, o que mostra que todos os genótipos, ao final do experimento, não haviam atingido seu peso máximo, ou seja, ainda estavam em fase de crescimento.
No modelo Logístico, assim como nos modelos de Gompertz e von Bertalanffy, o Carijó apresentou maior valor de "k", e o Caboclo o menor valor, indicando que o Carijó possui maior velocidade de crescimento em relação aos demais genótipos estudados.

Tabela 5. Parâmetros estimados para as curvas de crescimento dos genótipos de frangos tipo caipira segundo o modelo Logístico

\begin{tabular}{|c|c|c|c|c|c|c|c|}
\hline \multirow{2}{*}{ Genótipo } & \multicolumn{3}{|c|}{ Parâmetro } & \multirow{2}{*}{$\begin{array}{l}\mathrm{R}^{2} \\
(\%)\end{array}$} & \multirow{2}{*}{$\begin{array}{c}\text { DMA } \\
(\%)\end{array}$} & \multirow{2}{*}{$\begin{array}{c}\text { DPA } \\
(\%)\end{array}$} & \multirow{2}{*}{ IA } \\
\hline & $\mathrm{a}$ & $\mathrm{b}$ & $\mathrm{k}$ & & & & \\
\hline Caboclo & $\begin{array}{c}3861,8 \\
(3737,8 \mathrm{a} \\
3985,8)^{1}\end{array}$ & $\begin{array}{c}5,8718 \\
(5,6961 \mathrm{a} \\
6,0476) \\
\end{array}$ & $\begin{array}{c}0,0355 \\
(0,0341 \mathrm{a} \\
0,0369)\end{array}$ & 98,39 & 54,68 & 20,14 & 23,57 \\
\hline Carijó & $\begin{array}{c}4332,6 \\
(4224,9 \mathrm{a} \\
4440,3)\end{array}$ & $\begin{array}{c}6,2967 \\
(5,9862 \mathrm{a} \\
6,6073)\end{array}$ & $\begin{array}{c}0,0461 \\
(0,0441 \mathrm{a} \\
0,0481)\end{array}$ & 96,02 & 88,55 & 92,77 & 85,30 \\
\hline Colorpak & $\begin{array}{c}4822,8 \\
(4704,0 \mathrm{a} \\
4941,6) \\
\end{array}$ & $\begin{array}{c}6,5420 \\
(6,2265 \mathrm{a} \\
6,8574) \\
\end{array}$ & $\begin{array}{c}0,0458 \\
(0,0439 \mathrm{a} \\
0,0477) \\
\end{array}$ & 96,44 & 95,32 & 100,00 & 98,80 \\
\hline $\begin{array}{l}\text { Gigante } \\
\text { Negro }\end{array}$ & $\begin{array}{c}3817,4 \\
(3635,5 \mathrm{a} \\
3999,3) \\
\end{array}$ & $\begin{array}{c}5,8436 \\
(5,5410 \mathrm{a} \\
6,1462) \\
\end{array}$ & $\begin{array}{c}0,0373 \\
(0,0349 \mathrm{a} \\
0,0396) \\
\end{array}$ & 95,77 & 75,14 & 57,65 & 37,02 \\
\hline $\begin{array}{l}\text { Pesadão } \\
\text { Vermelho }\end{array}$ & $\begin{array}{c}4487,7 \\
(4365,7 \mathrm{a} \\
4609,7)\end{array}$ & $\begin{array}{c}6,3874 \\
(6,0701 \mathrm{a} \\
6,7047) \\
\end{array}$ & $\begin{array}{c}0,0448 \\
(0,0428 \mathrm{a} \\
0,0469)\end{array}$ & 95,92 & 95,07 & 97,37 & 96,52 \\
\hline $\begin{array}{l}\text { Pescoço } \\
\text { Pelado }\end{array}$ & $\begin{array}{c}4177,4 \\
(4062,9 \mathrm{a} \\
4291,8)\end{array}$ & $\begin{array}{c}6,0475 \\
(5,7663 \mathrm{a} \\
6,3286)\end{array}$ & $\begin{array}{c}0,0439 \\
(0,0420 \mathrm{a} \\
0,0459)\end{array}$ & 95,88 & 88,80 & 83,64 & 76,56 \\
\hline Tricolor & $\begin{array}{c}4577,8 \\
(4457,4 \mathrm{a} \\
4698,2)\end{array}$ & $\begin{array}{c}6,0510 \\
(5,8088 \mathrm{a} \\
6,2932)\end{array}$ & $\begin{array}{c}0,0423 \\
(0,0406 \mathrm{a} \\
0,0441)\end{array}$ & 96,75 & 100,00 & 74,31 & 77,56 \\
\hline
\end{tabular}

a-peso à idade adulta; b-constante de integração; k-taxa de maturidade; $\mathrm{R}^{2}$-coeficiente de determinação; DMA-desvio médio absoluto; DPA-desvio padrão assintótico; IA- índice assintótico.

${ }^{1}$ Intervalo de confiança a $95 \%$ entre parênteses.

Para o modelo Logístico, bem como para o modelo Gompertz, o ajuste do crescimento das aves do genótipo Caboclo apresentou os menores valores de DMA, DPA e IA, enquanto o Tricolor foi o que obteve maior valor de DMA no modelo Logístico. Quanto ao DPA e ao IA, o Colorpak apresentou maior valor. Com isso, pode-se afirmar que as aves do genótipo Caboclo se ajustaram melhor que as aves do genótipo Colorpak para o modelo Logístico.

O parâmetro " $k$ " é a relação entre a taxa de crescimento e o peso adulto do animal. Quanto maior o valor de "k", mais precoce é o animal
(Santos et al., 2007). Dessa maneira, pode-se verificar que o Carijó foi o mais precoce, e o Caboclo o mais tardio entre os genótipos estudados.

Vários autores também detectaram diferenças no crescimento de genótipos de frangos tipo caipira independentemente do modelo utilizado (Santos et al., 2005; Rizzi et al., 2013; Eleroğlu et al., 2014).

O coeficiente de determinação $\left(R^{2}\right)$ é outro critério utilizado para indicar a curva mais 
adequada para descrever o crescimento dos frangos tipo caipira nos modelos empregados. Os valores encontrados para todos os genótipos foram muito próximos de cem, portanto não foi possível, por esse critério, definir qual a curva que melhor se ajustou, ou seja, pelo $\mathrm{R}^{2}$ todos os modelos podem ser utilizados. Resultados semelhantes foram encontrados por Rizzi et al. (2013) e Eleroğlu et al. (2014) em trabalhos com frangos caipiras; Dzomba et al. (2011) e Mohammed (2015) em trabalhos com frangos de corte; Drumond et al. (2013) em trabalhos com codornas de corte.

Levando em consideração o IA, por se tratar de um critério de avaliação mais completo, pode-se observar que o melhor ajuste foi apresentado pela curva proposta por von Bertalanffy, sendo este o recomendado pelo presente estudo para descrever o padrão de crescimento das aves dos genótipos de frangos tipo caipira avaliados, conforme proposto por Dzomba et al. (2011) em trabalho com frangos de corte criados em sistemas intensivo e semi-intensivo. Em contrapartida, Eleroğlu et al. (2014) recomendaram o modelo Logístico, e Rizzi et al. (2013) recomendaram o modelo Gompertz para descrever o crescimento de frangos tipo caipira. Já segundo Sakomura e Rostagno (2007) e Mohammed (2015), o modelo de Gompertz foi o mais adequado para descrever o crescimento de frangos de corte, e Drumond et al. (2013) recomendaram os modelos de Gompertz e Logístico para descrição do crescimento de codornas de corte.

Em relação aos coeficientes de alometria, verificou-se que o crescimento alométrico foi heterogônico positivo para todos os cortes avaliados (Tab. 6; Fig. 1). Para o peso de peito, foi constatado $(\mathrm{P}<0,01) \quad$ crescimento heterogônico positivo $(\mathrm{b}>1)$ em todos os genótipos, indicando que o peso de peito apresentou desenvolvimento tardio em relação ao peso da carcaça, ou seja, a taxa de deposição do peso de peito é menor que a do peso da carcaça. As características de peso de coxas, sobrecoxas, pernas e asas também apresentaram tendência semelhante ao comportamento apresentado por peso de peito, com crescimento heterogônico positivo ( $b>1$, o que indica que peso de coxas, sobrecoxas, pernas e asas apresentam desenvolvimento tardio em relação ao peso da carcaça em todos os genótipos. Esse desenvolvimento pode ser explicado pelo fato de os genótipos tipo caipira possuírem crescimento considerado lento quando comparados ao frango de corte convencional.

Govaerts et al. (2000), em trabalho realizado com frangos de corte machos, também detectaram que o crescimento de peito, coxa, pernas, asa e gordura abdominal foi considerado tardio $(\mathrm{b}>1)$ em relação ao peso da carcaça. Zuidhof et al. (2014), igualmente, verificaram crescimento tardio de peito e coxa em três genótipos de frangos de corte. Entretanto, Danisman e Gous (2011) verificaram que o crescimento do peito de frangos de corte foi semelhante ao da carcaça, ou seja, isogônico.

Entre os cortes avaliados, as aves do genótipo Colopark apresentaram desenvolvimento mais precoce para todos os cortes quando comparado aos demais genótipos, conforme se pode observar pelos menores valores estimados para o parâmetro "b"(Tab. 6). Esse resultado pode ser explicado pelo fato de que essas aves têm um crescimento rápido, assim atingem mais cedo a idade adulta e, consequentemente, têm maior deposição proteica nos músculos, o que indica um bom potencial das aves para a produção de carne. O genótipo Tricolor, de forma geral, apresentou crescimento tardio dos cortes avaliados em relação aos demais, conforme pode ser observado pelos maiores valores estimados para o parâmetro "b".

Os valores dos coeficientes de determinação $\left(\mathrm{R}^{2}\right)$ para o estudo do crescimento alométrico foram altos, indicando que o ambiente de criação das aves foi o mais homogêneo possível e que, por isso, não interferiu de forma significativa no peso da carcaça e dos cortes, o que permite constatar, de forma significativa, a heterogonia positiva dos coeficientes da equação de Huxley. 
Tabela 6. Teste para crescimento isogônico $\left(\mathrm{H}_{0}: \mathrm{b}=1\right)$ dos cortes da carcaça em função do peso da carcaça, para os diferentes genótipos de frangos tipo caipira

\begin{tabular}{|c|c|c|c|}
\hline & Parâmetro b & $\mathrm{R}^{2}$ & $t_{\text {cal }}$ \\
\hline Genotıpo & & Peso & \\
\hline Carijó & 1,25914 & 0,9893 & $14,53393^{* *}$ \\
\hline Colorpak & 1,24388 & 0,9899 & $14,25365^{* *}$ \\
\hline Pesadão Vermelho & 1,26283 & 0,9913 & $16,31471^{* *}$ \\
\hline Pescoço Pelado & 1,27349 & 0,9920 & $13,70877^{* *}$ \\
\hline Tricolor & 1,28770 & 0,9894 & $12,42228^{* *}$ \\
\hline & & Pesc & \\
\hline Carijó & 1,03854 & 0,9986 & $7,258004^{* *}$ \\
\hline Colorpak & 1,03240 & 0,9969 & $4,169884^{* *}$ \\
\hline Pesadão Vermelho & 1,05352 & 0,9971 & $6,923674^{* *}$ \\
\hline Pescoço Pelado & 1,04498 & 0,9981 & $5,636591^{* *}$ \\
\hline Tricolor & 1,06887 & 0,9974 & $7,188935^{* *}$ \\
\hline & & Peso da & \\
\hline Carijó & 1,10237 & 0,9970 & $12,43864^{* *}$ \\
\hline Colorpak & 1,07109 & 0,9941 & $6,319111^{* *}$ \\
\hline Pesadão Vermelho & 1,08018 & 0,9915 & $5,895588^{* *}$ \\
\hline Pescoço Pelado & 1,09745 & 0,9982 & $11,89866^{* *}$ \\
\hline Tricolor & 1,12531 & 0,9940 & $8,244079^{* *}$ \\
\hline & & Peso & \\
\hline Carijó & 1,06906 & 0,9990 & $15,27876^{* *}$ \\
\hline Colorpak & 1,05076 & 0,9983 & $8,545455^{* *}$ \\
\hline Pesadão Vermelho & 1,06460 & 0,9971 & $8,292683^{* *}$ \\
\hline Pescoço Pelado & 1,07121 & 0,9993 & $14,86639^{* *}$ \\
\hline Tricolor & 1,09276 & 0,9989 & $14,72381^{* *}$ \\
\hline & & Pes & \\
\hline Carijó & 1,10132 & 0,9936 & $8,401327^{* *}$ \\
\hline Colorpak & 1,07749 & 0,9946 & $7,18165^{* *}$ \\
\hline Pesadão Vermelho & 1,13929 & 0,9899 & $8,911708^{* *}$ \\
\hline Pescoço Pelado & 1,11958 & 0,9918 & $6,752117^{* *}$ \\
\hline Tricolor & 1,12614 & 0,9932 & $7,776819^{* *}$ \\
\hline
\end{tabular}

** $\mathrm{P}<0,01 ; \mathrm{b}=$ coeficiente de crescimento relativo ou de alometria; $\mathrm{R}^{2}=$ coeficiente de determinação.

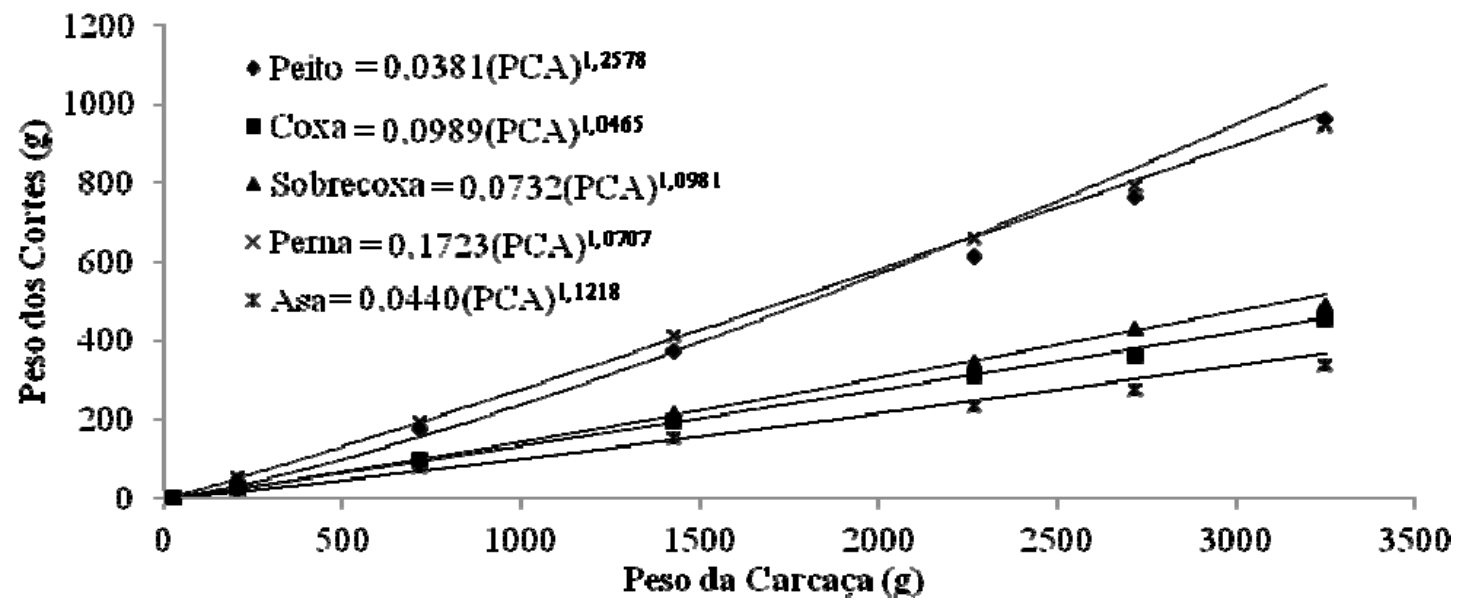

Figura 1. Desenvolvimento alométrico dos cortes (peito, coxa, sobrecoxa, perna e asa) em relação ao peso da carcaça (PCA) em frangos tipo caipira. 


\section{CONCLUSÕES}

Entre os modelos analisados, os modelos de Brody e Richards não se ajustaram à descrição do crescimento das aves tipo caipira. Os modelos de Gompertz, von Bertalanffy e Logístico se ajustaram bem ao crescimento dos genótipos estudados, e o modelo proposto por von Bertalanffy mostrou-se o mais adequado. $\mathrm{O}$ crescimento alométrico foi heterogônico positivo para todos os cortes avaliados em relação ao peso da carcaça nos genótipos de frangos tipo caipira, o que indica crescimento tardio dos cortes em relação ao peso da carcaça.

\section{AGRADECIMENTO}

Os autores agradecem o aporte financeiro e o apoio recebidos da Avifran, da Capes, do CNPq, da Fapemig e da Globoaves.

\section{REFERÊNCIAS}

ARAÚJO, R.O.; MARCONDES, C.R.; DAMÉ, M.C.F. et al. Classical nonlinear models to describe the growth curve for Murrah buffalo breed. Cien. Rural, v.42, p.520-525, 2012.

BRASIL. Ministério da Agricultura, Pecuária e Abastecimento. Departamento de Inspeção de Produtos de Origem Animal/ divisão de operações industriais. Registro do produto "frango caipira ou frango colonial" ou "frango tipo ou estilo caipira" ou "tipo ou estilo colonial”. Brasília, DF: MAPA, 2012. (Ofício Circular DOI/DIPOA $\mathrm{n}^{\circ} 02 / 2012$ de 01/02/2012).

DANISMAN, R.; GOUS, R.M. Effect of dietary protein on the allometric relationships between some carcass portions and body protein in three broiler strains. S. Afr. J. Anim. Sci., v.41, p.194208, 2011.

DRUMOND, E.S.C.; GONÇALVES, F.M.; VELOSO, R.C. et al. Curvas de crescimento para codornas de corte. Cienc. Rural, v.43, p.1872-1877, 2013.

DZOMBA, E.; DZOMBA-CENGIC, S.; MURATOVIC, S.; VASILJEVIC, B. Comparison of nonlinear models to describe growth of broiler chicken raised in confined or semi confined systems. In: INTERNATIONAL SCIENTIFICEXPERT CONFERENCE OF AGRICULTURE AND FOOD INDUSTRY, 22., 2011, Sarajevo. Proceedings... Saravejo: [s.n] 2011. p.28-31. (Resumo expandido).
ELEROĞLU, H.; YILDIRIM, A.; ŞEKEROĞLU, A. et al. Comparison of growth curves by growth models in slow-growing chicken genotypes raised the organic system. Int. J. Agric. Biol., v.16, p.529-535, 2014.

GOVAERTS T., ROOM G., BUYSE J. et al. Early and temporary quantitative food restriction of broiler chickens. 2. Effects on allometric growth and growth hormone secretion. Br. Poult. Sci, v.41 p.355-362, 2000.

HUXLEY, J.S. Problems of relative growth. London:Methuen, 1932. 577p.

MARCATO, S.M; SAKOMURA, N.K.; FERNANDES, J.B.K. et al. Crescimento e deposição de nutrientes nos órgãos de frangos de corte de duas linhagens comerciais. Rev. Bras. Zootec., v.39, p.1082-1091, 2010.

MENDONÇA, M.O.; SAKOMURA, N.K.; SANTOS, F.R. et al. Níveis de energia metabolizável para machos de corte de crescimento lento criados em semiconfinamento. Rev. Bras. Zootec., v.37, p.1433-1440, 2008.

MOHAMMED, F.A. Comparison of Three Nonlinear functions for Describing Chicken Growth Curves. Sci. Agric, v.9, p.120-123, 2015.

NAGIB NASCIMENTO, D.C.; SAKOMURA, N.K.; SIQUEIRA, J.C. et al. Exigências de lisina digestível para aves de corte da linhagem ISA Label criadas em semiconfinamento. Arq. Bras.Med. Vet. Zootec., v.61, p.1128-1138, 2009a.

NAGIB NASCIMENTO, D.C.; SAKOMURA, N.K.; SIQUEIRA, J.C. et al. Exigências de metionina + cistina digestível para aves de corte ISA Label criadas em semiconfinamento. Rev. Bras. Zootec., v.38, p.869-878, 2009 b.

PINHEIRO, S.R.F.; SAKOMURA, N.K.; NAGIB NASCIMENTO, D.C. et al. Níveis nutricionais de fósforo disponível para aves de corte ISA Label criadas em semiconfinamento. Rev. Bras. Zootec., v.40, p.361-369, 2011 a.

PINHEIRO, S.R.F.; SAKOMURA, N.K.; SIQUEIRA, J.C. et al. Níveis nutricionais de cálcio para aves de corte ISA Label criadas sob semiconfinamento. Arq. Bras. Med. Vet. Zootec., v.63, p.231-238, $2011 \mathrm{~b}$.

RATKOWSKY, D.A. Handbook of nonlinear regression models. New York and Basel, Marcel Dekker, 1990. 
RIZZI, C.; CONTIERO, B.; CASSANDRO, M. Growth patterns of Italian local chicken populations. Poult. Sci., v.92, p2226-2235, 2013.

ROSTAGNO, H.S., ALBINO, L.F.T., DONZELE, J.L. et al. Composição de alimentos e exigências nutricionais: tabelas brasileiras. Viçosa, M.G.: Universidade Federal de Viçosa, 2005. 141p.

SAKOMURA, N.K.; ROSTAGNO, H.S. Métodos de pesquisa em nutrição de monogástricos. Jaboticabal: Funep, 2007. 283p.

SANTOS, A.L; SAKOMURA, N.K.; FREITAS, E.R. et al. Estudo do crescimento, desempenho, rendimento de carcaça e qualidade de carne de três linhagens de frango de corte. Rev. Bras. Zootec., v.34, p.1589-1598, 2005.

SANTOS, V.B.; FREITAS, R.T.F.; SILVA, F.F. et al. Avaliação de curvas de crescimento morfométrico de linhagens de tilápia do Nilo (Oreochromis niloticus). Cienc. Agrotec., v.31, p.1486-1492, 2007.
SARMENTO, J.L.R.; REGAZZI, A.J.; SOUZA, W.H. et al. Estudo da curva de crescimento de ovinos Santa Inês. Rev. Bras. Zootec., v.35, p.435-442, 2006.

SILVA, M.A.N. ; HELLMEISTER FILHO, P.; ROSÁRIO, M.F. et al. Adaptação de linhagens de galinhas para corte ao sistema de criação semi-intensivo. Rev. Bras. Cienc. Avic., v.4, p.219-225, 2002.

STATISTICAL analysis systems: user's guide. Version 9.1, Cary: SAS, 2002. v.2, 1052p.

THOLON P.; QUEIROZ, S.A. Modelos matemáticos utilizados para descrever curvas de crescimento em aves aplicados ao melhoramento animal. Cienc. Rural, v.39, p.2261-2269, 2009.

ZUIDHOF, M.J.; SCHNEIDER, B.L.; CARNEY, V.L. et al. Growth, efficiency, and yield of commercial broilers from 1957, 1978, and 2005. Poult. Sci., v.93, p1-13, 2014. 